\title{
Frequency-Hopping Based SCMA for Massive Connectivity in Multi-cell Networks
}

\author{
Qi Zeng ${ }^{\dagger}, \quad$ Zilong Liu ${ }^{\ddagger}, \quad$ Xing Liu ${ }^{\dagger}, \quad$ Jun Zhong ${ }^{\dagger}, \quad$ Pei Xiao ${ }^{\$}$ \\ $\dagger$ College of Electrical Engineering, Sichuan University, Chengdu, China \\ $\ddagger$ School of Computer Science and Electronics Engineering, University of Essex, UK \\ $\$ 5$ GIC and 6GIC, Institute for Communication Systems, University of Surrey, UK \\ Emails: qzeng1@hotmail.com, zilong.liu@essex.ac.uk, liuxing4@126.com, zhongjun55@163.com, p.xiao@surrey.ac.uk
}

\begin{abstract}
Sparse code multiple-access (SCMA) is an emerging technique to support massive connectivity in 5G networks and beyond. In SCMA transmissions, some resource-blocks may undergo certain contamination due to deep fading and/or jamming attacks, thus leading to severe performance degradation over such contaminated ones. Besides, the current SCMA infrastructure is normally deployed in single-cell. To deploy the SCMA into multicell networks under contaminated/jamming channels, we propose a novel frequency-hopping based SCMA (FH-SCMA) for quasisynchronous multi-cell networks, in which the entire subcarrierchannels of every codeword keep hopping over the multiple resource-blocks according certain hopping pattern. We propose and design a pseudo-randomly orthogonal hopping pattern to adapt to the specific requirements of quasi-synchronous FHSCMA multi-cell networks. Our analysis and simulation results indicate that the proposed FH-SCMA leads to both improved user capacity and error-rate performance, whilst remaining resilient to the inter-cell interference.
\end{abstract}

Index Terms-Sparse code multiple access, frequency hopping, hopping pattern design, multi-cell networks

\section{INTRODUCTION}

In the fifth generation cellular (5G) networks and beyond, sparse code multiple-access (SCMA) [1], [2] is widely regarded as one of the most promising techniques to support exponential growth of communication devices (users) over the limited and congested spectral resource-blocks (RBs) [3], [4]. These devices which widely present in a broad range of vertical industries (such as smart homes/cities, factories of future, connected autonomous vehicles) are densely deployed to form the heterogenous multi-cell networks [5], as illustrated in Fig.1. In multi-cell networks, each micro-cell is comprised of massive devices which are connected by SCMA, while multiple micro-cells are overlapped geographically resulting to the severe inter-cell interference if spectral resource is not properly managed.

As a non-orthogonal access scheme, SCMA is characterized by its sparse codebooks which enable efficient multiuser detection and multi-user interference mitigation with the aid of message passing algorithm. Despite numerous research attempts on SCMA in the literature [6]-[11], it is mostly assumed that every user's codebook is sent over a few fixed subcarriers for consecutive transmissions of SCMA blocks. Thus, excellent error rate performances may not be attained when some subcarriers suffer from deep fading or jamming

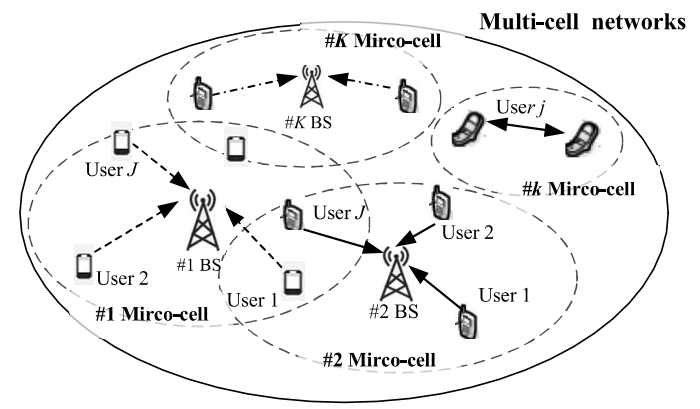

Fig. 1. An illustration of multi-cell networks with massively connected communication devices.

attacks. In addition, existing works in [6], [8]-[11] just focus on SCMA codebooks design for single-cell network.

To address the aforementioned issue, frequency-hopping based SCMA (FH-SCMA) has been studied in recent years [12], [13], where FH is helpful for avoiding the effect of channel contaminations by changing the instant channel according certain hopping pattern and supporting multi-cell access. The FH technique is a potential solution to the interference mitigation in the 5G multi-cell networks and beyond [14], [15]. In [12], optimal FH patterns for FH-SCMA are proposed by using quadratic congruence, but such a FH-SCMA scheme is just applicable to the single-cell uplinks. A new FH-SCMA for multi-cell networks has been developed in [13]. The key idea of [13] is to hop the entire RB of SCMA codebook over a wider range of spectrum so as to avoid certain consecutively contaminated channels; however in [13], a random hopping pattern is employed in FH-SCMA, which leads to the amount of inter-cell interference due to the existence of frequency-hits.

This paper aims to address the aforementioned problems in FH-SCMA based multi-cell networks on effective design of $\mathrm{FH}$ patterns to attain significantly improved error rate performance. As shown in Fig. 1, within each micro-cell, multiple users form an SCMA group to connect with the base station (BS). Among multi-cells, certain properly designed hopping patterns are assigned to different micro-cells ${ }^{1}$. It is noted that, in this paper, the quasi-synchronous access

\footnotetext{
${ }^{1}$ Thus, each hopping pattern is also referred to as a cell address
} 
mechanism is considered, which is a most piratical case for multi-cells belonging to different wireless applications. Our proposed hopping pattern possesses the promising merit of orthogonality for quasi-synchronization. By employing such a hopping pattern into FH-SCMA, the simulation and theoretical results show that it can efficiently reduce the effect of contaminated channels, whilst completely avoiding the intercell interference for multi-cell networks.

\section{SySTEM MODEL}

In this section, we firstly recall the traditional single-cell SCMA. Then the critical issues in the current SCMA can be straightway extracted. In order to address these issues, the idea of the new FH-SCMA is proposed for multi-cell netowrks, which is extended from the traditional one.

\section{A. Traditional SCMA and problem descriptions}

We consider a traditional $(K \times J)$ SCMA in the single-cell network, where $J$ users are multiplexed to connect to BS over $K$ RBs. The SCMA codebooks are properly designed, denoted by

$$
\begin{array}{r}
\mathbb{X}=\left\{\mathbf{X}_{1}, \mathbf{X}_{2}, \cdots, \mathbf{X}_{J}\right\}, \\
\mathbf{X}_{j}=\left[\mathbf{x}_{1}^{(j)}, \mathbf{x}_{2}^{(j)}, \cdots, \mathbf{x}_{M}^{(j)}\right],
\end{array}
$$

where each codebook $\mathbf{X}_{j}$ which may be arranged as a $(K \times M)$ matrix, satisfies $\operatorname{Tr}\left(\mathbf{X}_{j} \mathbf{X}_{j}^{H}\right)=M$. The column vector $\mathbf{x}_{m}^{(j)}$ with $(K \times 1)$ entries is called a codeword with low-density, which is denoted as $\mathbf{x}_{m}^{(j)}=\left(x_{m, 1}^{(j)}, x_{m, 2}^{(j)}, \cdots, x_{m, K}^{(j)}\right)^{\mathrm{T}}$. At the transmitter, the SCMA coder of user $j$ selects a codeword from $\mathbf{X}_{j}$ (i.e., a column-vector $\mathbf{x}_{m}^{(j)}$ ) based on the instantaneously $M$-ary transmitted symbol $m \in\{1,2, \cdots, M\}$. The designs of the SCMA codebook can be found in [8], [9], which need the delicate mathematical tricks but out of the scope of this paper.

Assuming the channel-gains associated to the $j$-th user to be $\mathbf{h}_{j}=\left\{h_{j, 1}, h_{j, 2}, \cdots, h_{j, K}\right\}$ according to these preassigned $K$ RBs. Given the transmitted $M$-ary symbol $m \in$ $\{1,2, \cdots, M\}$ which is encoded as SCMA codeword $\mathbf{x}_{m}^{(j)}$, the $K$-dimensional received signal y of $J$ users can be expressed as

$$
\mathbf{y}=\sqrt{P / J} \sum_{j=1}^{J} \operatorname{diag}\left(\mathbf{h}_{j}\right) \mathbf{x}_{m}^{(j)}+\mathbf{n},
$$

where $P$ denotes the total transmit power and assume uniform power allocation among $J$ users. $\mathbf{n}$ is a $(K \times 1)$ vector with each element following the additional white Gaussian noise (AWGN).

For convenient explanation, an example of transmitted SCMA codewords of the $j$-th user with $K=4 \mathrm{RBs}$ is demonstrated in Fig. 2. In this figure, the $n$-th $M$-ary symbol $m$ has been encoded as the SCMA codeword with low density $\mathbf{x}_{m}^{(j)}(n)=\left(x_{m, 1}^{(j)}(n), x_{m, 2}^{(j)}(n), x_{m, 3}^{(j)}(n), x_{m, 4}^{(j)}(n)\right)^{\mathrm{T}}$. The nonzero entries of SCMA codeword are transmitted over the fixed RBs, i.e., over the 2nd and 4th RBs in Fig. 2. In the practical wireless communications, the entire or the part of

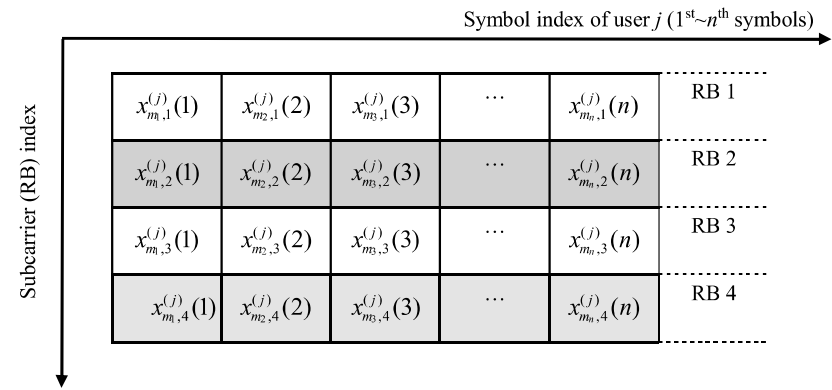

Fig. 2. The typical SCMA symbols transmitted over 4 RBs $(K=4$ subcarrier-channels).

$\mathrm{RBs}$ in $\mathbf{h}_{j}$ may be contaminated by deep fading or jamming attacks. For example as shown in Fig. 2, when the 2nd and 4th RBs are contaminated by jammer, this user will continuously undergo the severe performance degradations. The current SCMA system is normally deployed in the single-cell network, but it is a more interesting work to extend the single-cell SCMA to the multi-cell scenario in the future.

\section{B. FH-SCMA in multi-cell networks}

Following the conventional SCMA, we adopt the FH pattern as the micro-cells address to allow the multi-cells access simultaneously; besides, the FH employed in SCMA can help to avoid the contaminated RBs. In such a proposed FH-SCMA multi-cell networks, all users within the single micro-cell are easy synchronized with intra-cell users; meanwhile, intercells are assumed to be quasi-synchronous access. Some key notations for the proposed FH-SCMA system are given firstly.

- $\mathbb{F}=\left\{f_{1}, f_{2}, \cdots, f_{K_{R B}}\right\}$ : the available spectral RBs set with size $K_{R B}$

- $\mathbb{H}=\left\{h_{1}, h_{2}, \cdots, h_{K_{R B}}\right\}$, where the channel gain $h_{i}$ is corresponding to the RB $f_{i}$ in $\mathbb{F}$.

- $\mathbb{F}_{M C}^{(i)}$ : a RBs subset selected from $\mathbb{F}$ and its size $\left\|\mathbb{F}_{M C}^{(i)}\right\|=K, i=1,2, \cdots$. The set $\mathbb{F}_{M C}^{(i)}$ is referred as a RB-group.

- $J$ : The number of users accommodated in each microcell. These $J$ users are referred as a user-group.

Based on aforementioned notations, the number of orthogonal RB-groups generated over the RBs set $\mathbb{F}$ is eqal to $Q=\left\lfloor K_{R B} / K\right\rfloor$. These RB-groups can be represented as

$\mathbb{F}_{M C}=\left\{\mathbb{F}_{M C}^{(0)}, \mathbb{F}_{M C}^{(1)}, \cdots, \mathbb{F}_{M C}^{(Q-1)}\right\}, \mathbb{F}_{M C}^{(i)} \cap \mathbb{F}_{M C}^{(j)}=\emptyset, i \neq j$.

For convenient analysis, we assume that the RB-group $\mathbb{F}_{M C}^{(i)}$ consists of $K$ consecutive RBs and undergos the constant contamination. The different RB-groups may have the various levels of contamination due to the existence of the deep fading or jamming attacks. Thus, for these $Q$ RB-groups, the RBgroups channel-gains are denoted as

$$
\mathbb{H}^{\prime}=\left\{h_{0}^{\prime}, h_{1}^{\prime}, \cdots, h_{Q-1}^{\prime}\right\},
$$

where $h_{i}^{\prime}$ is the RB-group channel-gain corresponding to the RB-group $\mathbb{F}_{M C}^{(i)}$. By absorbing the effect of contamination into 


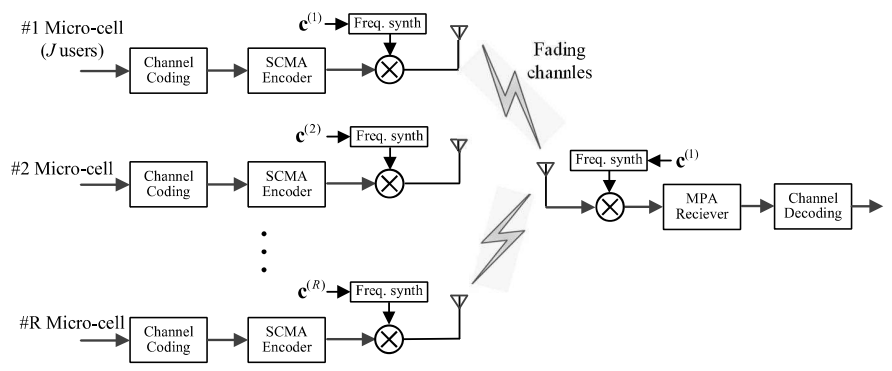

Fig. 3. The transceiver model of FH-SCMA system in multi-cell networks.

Rayleigh fading channel, the probability-of-density (pdf) of the amplitude of channel-gain $r_{i}=\left\|h_{i}^{\prime}\right\|$ can be written as follows with various parameters $\sigma_{i}$.

$$
p_{r}\left(r_{i}\right)=\frac{r_{i}}{\sigma_{i}^{2}} \exp \left(-\frac{r_{i}^{2}}{2 \sigma_{i}^{2}}\right), i=0,1, \cdots, Q-1
$$

where the mean square value $\overline{r_{i}^{2}}=2 \sigma_{i}^{2}$.

Based on multiple RB-groups $\mathbb{F}_{M C}$, the FH-SCMA transceiver model for multi-cells is proposed, as shown in Fig. 3. In the proposed FH-SCMA, a user-group is multiplexed in the micro-cell by using the traditional $(K \times J)$ SCMA codebook. Following by the SCMA encoder, the instantaneous RB-group for each multi-cell is hopped over the set $\mathbb{F}_{M C}$ according to the pre-signed hopping sequence $\mathbf{c}^{(r)}$. The FH sequence $\mathbf{c}^{(r)}$ assigned to the $r$-th micro-cell can be represented as

$$
\mathbf{c}^{(r)}=\left(c_{0}^{(r)}, c_{1}^{(r)}, \cdots, c_{L-1}^{(r)}\right),
$$

where $L$ denotes the length of hopping sequence. The active RB-group hopping paradigm of FH-SCMA in one micro-cell is illustrated in Fig. 4. In this figure, the active RB-group $(K=4)$ is randomly changed over the available RBs set $\mathbb{F}_{M C}$ following the pre-signed $\mathrm{FH}$ sequence $\mathbf{c}^{(r)}$. In the following analysis, one $M$-ary symbol is send over one hopping interval.

Again, we remind that the perfect synchronization and quasi-synchronization have been performed within single micro-cell and among the micro-cells, respectively. Thus, the multiplexed signal of $R$ micro-cells over the $l$-th symbol duration can be written as

$$
\mathbf{y}_{l}=\sqrt{P / J} \sum_{r=1}^{R} \sum_{j=1}^{J} \operatorname{diag}\left(\mathbf{h}_{j}^{\left(c_{l+\tau_{r}}^{(r)}\right)}\right) \mathbf{x}_{m}^{(r, j)}\left(l+\tau_{r}\right)+\mathbf{n},
$$

where $\tau_{r}$ denotes the access delay among the multiple microcells. The addition $l+\tau_{r}$ in subscript performs modulo $L$. $\mathbf{x}_{m}^{(r, j)}(\cdot)$ denotes the codeword sent from the $j$-th user in the $r$-th micro-cell. $\mathbf{h}_{j}^{(c)} \in \mathbb{H}^{\prime}$ are the channel gains of the RBgroup $\mathbb{F}_{M C}^{(c)}$ according to the pre-signed $\mathrm{FH}$ sequence $\mathbf{c}^{(r)}$. For the quasi-synchronous access mechanism, we have $\left|\tau_{r}\right| \leq$ $Z T<<L T$, where $Z$ is a small integer and $T$ is the duration of one hopping interval.

In the receiver of desired micro-cell (i.e., $r=1$ ), the acess delay is assumed to $\tau_{1}=0$. The received signals are orderly

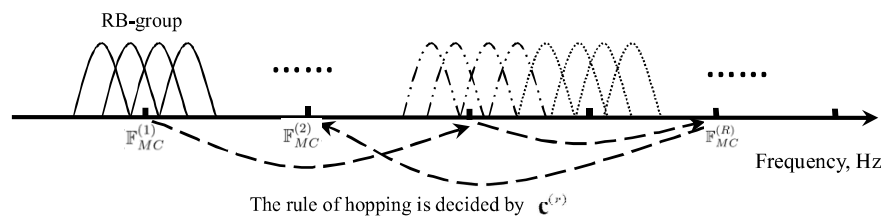

Fig. 4. The RB-group hopping paradigm of FH-SCMA.

processed through dehopper, MPA decoder and detector as shown in Fig. 3.

Throughout the aforementioned analysis, we find that the properties of FH pattern is the critical component to approach the target of channel jamming/contamination mitigation. These properties should meet the following requirements.

- The hopping pattern should be orthogonal for quasisynchronous multi-cell networks, which can completely avoid the inter-cell interference.

- The number of each RB-group appearing in FH patterns should be identical, which help to randomize the effect of contaminated channels.

- The randomness of hopping pattern should be satisfied, which guarantees the capability of anti-jamming.

\section{Design of FH PATtern AND EXAMPLE}

In this section, a construction algorithm of the hopping pattern meeting the aforementioned properties is proposed. Then we present an example of the proposed FH pattern.

\section{A. Construction algorithm of FH pattern}

Given $R$ micro-cells overlapped in the networks, where the maximal access delay among these micro-cells is $Z$, the FH pattern for quasi-synchronization FH-SCMA networks is denoted as

$$
\begin{aligned}
& \mathbb{C}=\left\{\mathbf{c}^{(1)}, \mathbf{c}^{(2)}, \cdots, \mathbf{c}^{(R)}\right\}, \\
& \mathbf{c}^{(r)}=\left(c_{0}^{(r)}, c_{1}^{(r)}, c_{2}^{(r)}, \cdots, c_{L-1}^{(r)}\right), c_{l}^{(r)} \in \tilde{\mathbb{F}}_{M C} .
\end{aligned}
$$

where $\tilde{\mathbb{F}}_{M C}$ is the central frequency set with regard to the available RB-groups set $\left\{\mathbb{F}_{M C}^{(0)}, \mathbb{F}_{M C}^{(1)}, \cdots, \mathbb{F}_{M C}^{(Q-1)}\right\}$, which is denoted as $\tilde{\mathbb{F}}_{M C}=\left\{f_{0}, f_{1}, f_{2}, \cdots, f_{Q-1}\right\}$.

The orthogonal hopping pattern over these RB-groups $\tilde{\mathbb{F}}_{M C}$ through the following steps.

Step 1: We construct a matrix $\mathbb{E}_{0}$ of dimensions $(R \times(Z+$ 1)) with non-repeating element over the set $\tilde{\mathbb{F}}_{M C}$. Thus, $\mathbb{E}_{0}$ is written as

$$
\mathbb{E}_{0}=\left[\mathbf{e}_{0}, \mathbf{e}_{1}, \cdots, \mathbf{e}_{Z}\right]=\left[\begin{array}{cccc}
e_{0}^{0} & e_{0}^{1} & \cdots & e_{0}^{Z} \\
e_{1}^{0} & e_{1}^{1} & \cdots & e_{1}^{Z} \\
\vdots & \vdots & \ddots & \vdots \\
e_{R-1}^{0} & e_{R-1}^{1} & \cdots & e_{R-1}^{Z}
\end{array}\right]
$$

where $\mathbf{e}_{z}$ denotes a column-vector, and there does not exist any same element in $\mathbb{E}_{0}$. To achieve optimal properties, we consider a prime integer $R$ in this paper.

Step 2: Given $Z+1$ non-repeating positive integers $\left\{a_{z} \mid z=\right.$ $0,1,2, \cdots, Z\}$, where $a_{z} \in\{1,2, \cdots, R-1\}$ and $a_{i} \neq a_{j}$ if 
$i \neq j$. Operating the cyclic $a_{z}$-digit(s) shifts on $\mathbf{e}_{z}$ respectively, then we get

$$
\begin{gathered}
\tilde{\mathbf{e}}_{z}=\mathfrak{S}\left(a_{z} \mid \mathbf{e}_{z}\right) \triangleq\left(e_{a_{z}}^{z}, e_{a_{z}+1}^{z}, \cdots, e_{R-1}^{z}, e_{0}^{z}, e_{1}^{z}, \cdots, e_{a_{z}-1}^{z}\right)^{T}, \\
z=0,1,2, \cdots, Z
\end{gathered}
$$

where $\mathfrak{S}\left(a_{z} \mid \mathbf{e}_{z}\right)$ denotes the cyclic $a_{z}$-digit(s) shifts operation on $\mathbf{e}_{z}$. Then, based on (12), a new matrix is obtained as

$$
\mathbb{E}_{1}=\left[\mathfrak{S}\left(a_{0} \mid \mathbf{e}_{0}\right), \mathfrak{S}\left(a_{1} \mid \mathbf{e}_{1}\right), \cdots, \mathfrak{S}\left(a_{Z} \mid \mathbf{e}_{Z}\right)\right]
$$

Step 3: Further we take the specific cyclic shift operations as following equations on each column vector of $\mathbb{E}_{0}$.

$$
\left\{\begin{array}{c}
\mathbb{E}_{2}=\left[\mathfrak{S}\left(2 a_{0} \mid \tilde{\mathbf{e}}_{0}\right), \mathfrak{S}\left(2 a_{1} \mid \tilde{\mathbf{e}}_{1}\right), \cdots, \mathfrak{S}\left(2 a_{Z} \mid \tilde{\mathbf{e}}_{Z}\right)\right], \\
\vdots \\
\mathbb{E}_{r}=\left[\mathfrak{S}\left(r a_{0} \mid \tilde{\mathbf{e}}_{0}\right), \mathfrak{S}\left(r a_{1} \mid \tilde{\mathbf{e}}_{1}\right), \cdots, \mathfrak{S}\left(r a_{Z} \mid \tilde{\mathbf{e}}_{Z}\right)\right], \\
\vdots \\
\mathbb{E}_{R-1}=\left[\mathfrak{S}\left((R-1) a_{0} \mid \tilde{\mathbf{e}}_{0}\right), \mathfrak{S}\left((R-1) a_{1} \mid \tilde{\mathbf{e}}_{1}\right), \cdots, \mathfrak{S}\left((R-1) a_{Z} \mid \tilde{\mathbf{e}}_{Z}\right)\right] .
\end{array}\right.
$$

Step 4: Cascading all matrix $\left\{\mathbb{E}_{r} \mid r=0,1, \cdots, R-1\right\}$ together, then we get a new matrix as follows.

$$
\mathbb{C}=\left[\mathbb{E}_{0}, \mathbb{E}_{1}, \cdots, \mathbb{E}_{R-1}\right]
$$

It is easy to obtain that the matrix $\mathbb{C}$ has the dimension of $(R \times R(Z+1))$. Finally, $\mathbb{C}$ can be rewritten in the row-manner as follows.

$$
\mathbb{C}=\left[\begin{array}{c}
\mathbf{c}^{(1)} \\
\mathbf{c}^{(2)} \\
\vdots \\
\mathbf{c}^{(R)}
\end{array}\right]=\left\{\begin{array}{cccc}
\left(c_{0}^{(1)},\right. & c_{1}^{(1)}, & \cdots & \left.c_{L-1}^{(1)}\right) \\
\left(c_{0}^{(2)},\right. & c_{1}^{(2)}, & \cdots & \left.c_{L-1}^{(2)}\right) \\
\vdots & \vdots & \ddots & \vdots \\
\left(c_{0}^{(R)},\right. & c_{1}^{(R)}, & \cdots & \left.c_{L-1}^{(R)}\right)
\end{array}\right\}
$$

The set $\mathbb{C}$ is the proposed FH pattern with the size $R$ and length $L=R(Z+1)$, and it meets orthogonal when the access delay is less than $Z$. The size of the available RB-groups set $\tilde{\mathbb{F}}_{M C}$ is equal to $Q=R(Z+1)$.

\section{B. Example}

Given the number of micro-cells $R=5$ and the maximal access delay $Z=2$, we intend to construct the orthogonal hopping pattern over the RB-groups set $\tilde{\mathbb{F}}_{M C}=$ $\left\{f_{0}, f_{1}, f_{2}, \cdots, f_{14}\right\}$.

Firstly, we construct the matrix $\mathbb{E}_{0}$ according to Step 1.

$$
\mathbb{E}_{0}=\left[\begin{array}{ccc}
0, & 2, & 10 \\
3, & 4, & 7 \\
14, & 13, & 9 \\
6 & 12, & 1 \\
8, & 5, & 11
\end{array}\right]
$$

where the entry $i$ denotes the central frequency of RB-group $f_{i}$ for short.
Then, setting the parameters $a_{0}=1, a_{1}=3$ and $a_{2}=2$, multiple matrices are obtained as follows via the specific cyclic shift operations as shown in (14).

$$
\mathbb{E}_{1}=\left[\begin{array}{ccc}
3, & 12, & 9 \\
14, & 5, & 1 \\
6, & 2, & 11 \\
8 & 4, & 10 \\
0, & 13, & 7
\end{array}\right], \cdots, \mathbb{E}_{4}=\left[\begin{array}{ccc}
8, & 13, & 1 \\
0, & 12, & 11 \\
3, & 5, & 10 \\
14 & 2, & 7 \\
6, & 4, & 9
\end{array}\right]
$$

Cascading all matrices of (17) and (18), the orthogonal hopping pattern for access delay $Z=2$ is obtained as follows according to Step 4.

$$
\begin{aligned}
& \mathbb{C}=\left[\mathbb{E}_{0}, \mathbb{E}_{1}, \mathbb{E}_{2}, \mathbb{E}_{3}, \mathbb{E}_{4}\right] \\
& =\left\{\begin{array}{l}
(0,2,10,3,12,9,14,4,11,6,5,7,8,13,1) \\
(3,4,7,14,5,1,6,13,10,8,2,9,0,12,11) \\
(14,13,9,6,2,11,8,12,7,0,4,1,3,5,10) \\
(6,12,1,8,4,10,0,5,9,3,13,11,14,2,7) \\
(8,5,11,0,13,7,3,2,1,14,12,10,6,4,9)
\end{array}\right\} .
\end{aligned}
$$

It is easy to obtain that $R=5$ and $L=15$. Then, we will check the number of mutual/auto frequency-hits over the entire sequence length, i.e., Hamming cross- and auto- correlations $\left(H_{c}(\tau)\right.$ and $\left.H_{a}(\tau)\right)$. Taking $\mathbf{c}^{(1)}$ as an example, the values of $H_{c}(\tau)$ and $H_{a}(\tau)$ are computed as

$$
\begin{aligned}
& H_{c}\left(\tau \mid \mathbf{c}^{(1)}, \mathbf{c}^{(2)}\right)=(0,0,0,5,0,0,5,0,0,5,0,0,0,0,0), \\
& H_{c}\left(\tau \mid \mathbf{c}^{(1)}, \mathbf{c}^{(3)}\right)=(0,0,0,5,0,0,0,0,0,5,0,0,5,0,0), \\
& H_{c}\left(\tau \mid \mathbf{c}^{(1)}, \mathbf{c}^{(4)}\right)=(0,0,0,0,0,0,5,0,0,5,0,0,5,0,0), \\
& H_{a}\left(\tau \mid \mathbf{c}^{(1)}\right)=(15,0,0,0,0,0,0,0,0,0,0,0,0,0,0),
\end{aligned}
$$

where $0 \leq \tau \leq 15, \tau \in \mathbb{Z}^{+}$. From the obtained $H_{c}(\tau)$ and $H_{a}(\tau)$, we find that the FH pattern $\left\{\mathbf{c}^{(i)}\right\}$ is orthogonal when $|\tau| \leq Z$, that is, $H_{c}(\tau) \equiv 0$ and $H_{a}(\tau \neq 0) \equiv 0$. Such properties imply that the inter-cell interference can be completely canceled as the micro-cells perform quasi-synchronization.

\section{Simulation AND Discussions}

In this section, the error-rates of quasi-synchronous $\mathrm{FH}$ SCMA multi-cell networks employing with the proposed hopping pattern under the contaminated channels are investigated by Matlab simulations. In the following simulations, we assume that $15 \mathrm{RB}$-groups are shared by 5 micro-cells $(R=5)$, and each micro-cell employs $(K \times J)=(4 \times 6)$ SCMA codebook to accommodate massive users. The maximal access delays among micro-cells are less than $2 T$.

The BERs performance of multiple micro-cells employing with FH-SCMA and the traditional SCMA are compared in Fig. 5, where one RB-group suffers from deep contamination with the parameter $\overline{r^{2}}=0.5$ and the other RB-groups are assumed to be flat Rayleigh fading with the parameter $\overline{r^{2}}=1$. For the traditional SCMA, the micro-cell operating on the RBgroup with good quality attains the lowest BER (labeled by the dash red line); while the micro-cell operating over the deeply contaminated RB-group has the worst-case of BER (labeled by the dash black line). Thus for the traditional SCMA deploying in multi-cells networks, different micro-cells 


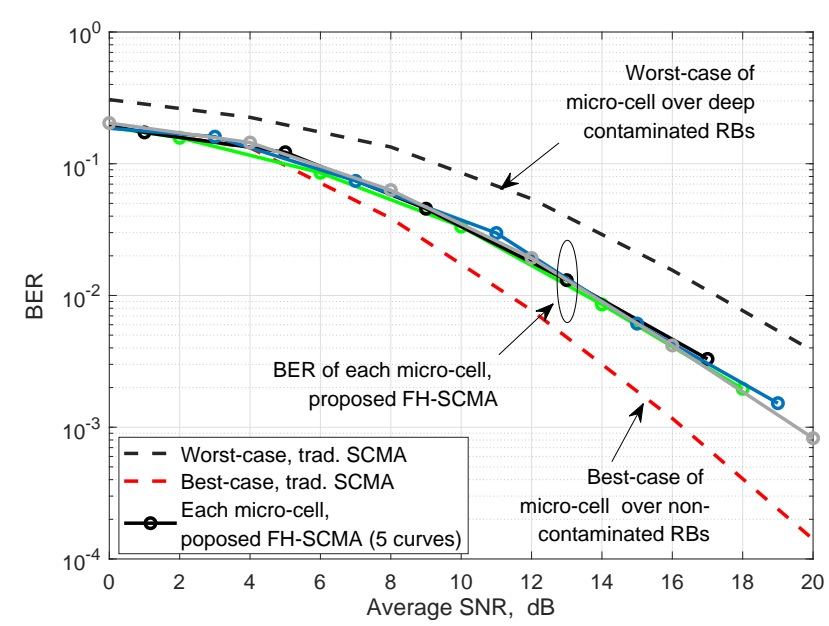

Fig. 5. The BER comparisons of multiple micro-cells employing FH-SCMA and the traditional SCMA.

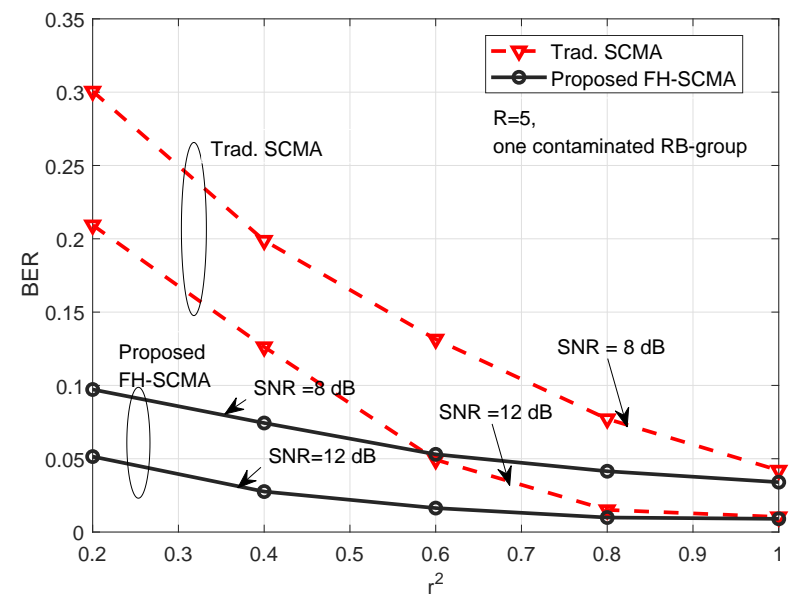

Fig. 6. The effect of RB-group contamination parameter $\overline{r^{2}}$ on the BER of the FH-SCMA.

have the unbalanced BER performance. By imposing FH into SCMA, all micro-cells have the similar BER behavior, of which curve lies between the worst- and best- case curves. The reason of this phenomenon is that $\mathrm{FH}$ technique can randomize the effect of deeply contaminated RB-groups among the all micro-cells, so that all micro-cells approach the optimal BER performance.

The effect of RB-group contamination parameters $\overline{r^{2}}$ on the BER of the FH-SCMA is presented in Fig. 6, where one RBgroup is assumed to be contaminated with the different SNRs. The smaller $\overline{r^{2}}$ refers to the more severer contamination that the RB-group undergoes. Observed from this figure, the BER decreases with the increasing $\overline{r^{2}}$, and SCMA system with FH technique significantly outperforms that without $\mathrm{FH}$.

The BERs of SCMA-FH in multi-cell networks under various RBs conditions are shown in Fig. 7. The two cases of RBs conditions are investigated, that is, one contaminated RBgroup $\left(\overline{r^{2}}=\{0.5,0.8,0.9\}\right)$ and two contaminated RB-groups

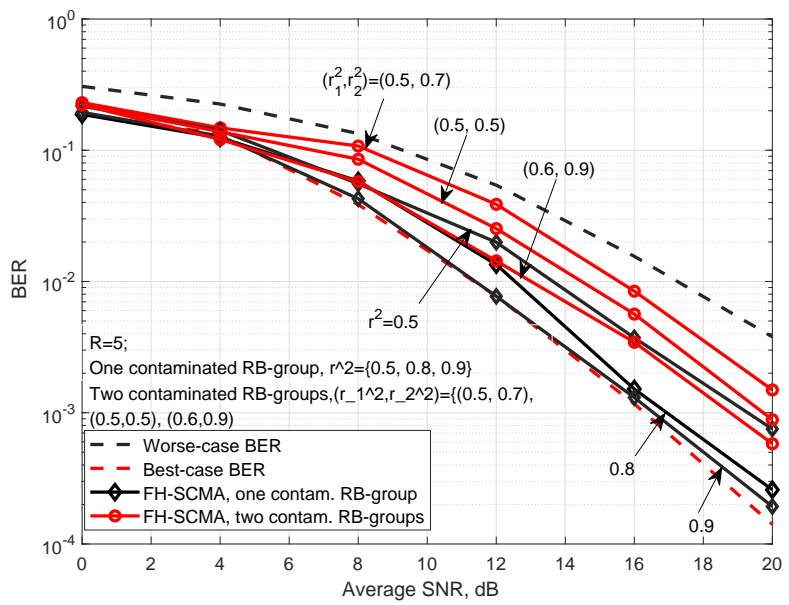

Fig. 7. The BERs of SCMA-FH system in multi-cell networks over various $\mathrm{RB}$-groups conditions.

$\left(\left(\overline{r_{1}^{2}}, \overline{r_{2}^{2}}\right)=\{(0.5,0.7),(0.5,0.5),(0.6,0.9)\}\right)$. Observed from this figure, all BERs of the proposed FH-SCMA lie in the region between the best-case and the worst-case curves. The FH technique can offer the performance benefits for various RB conditions. Particularly, for the case of two contaminated RB-groups, the proposed FH-SCMA can still alleviate the affect of contaminated RBs on BER performance. Thus, it is verified that the proposed FH-SCMA can utilize the FH technique to significantly suppress the contamination of RBs.

\section{CONCLUSIONS}

In traditional SCMA systems, the performance may be deteriorated when SCMA codewords are send over the fixed RBs that suffer from the contamination due to the multi-path fading and jamming attacks. In addition, the current SCMA infrastructure is normally deployed in the single-cell network but not suitable for the multi-cell scenario. In this paper, the FH technique is imposed in SCMA for the quasi-synchronous multi-cell networks. By properly designing a hopping pattern, multiple quasi-synchronous micro-cells can simultaneously operate without any inter-cell interference; meanwhile all multi-cell approach to the optimal BER performance by randomizing the effect of the contaminated RBs. Noted that this paper just concentrates on the FH-SCMA multi-cell infrastructure and hopping pattern designs, the theoretical error-rate of such networks will be investigated in the future work.

\section{ACKNOWLEDGMENT}

The work was supported by National Science Foundation of China under Grants 61701328 and 61901288, and in part by Foundation of Science and Technology on Communication Security Laboratory of China under Grant No. 6142103200106.

\section{REFERENCES}

[1] H. Nikopour and H. Baligh, "Sparse code multiple access," in IEEE 24th Annual International Symposium on Personal Indoor and Mobile Radio Communications (PIMRC), 2013, pp. 332-336. 
[2] M. Taherzadeh, H. Nikopour, A. Bayesteh, and H. Baligh, "SCMA codebook design," in IEEE 80th Vehicular Technology Conference (VTC2014Fall), 2014, pp. 1-5.

[3] L. Dai, B. Wang, Y. Yuan and et al., "Non-orthogonal multiple access for 5G: solutions, challenges, opportunities, and future research trends," IEEE Commun. Mag., vol. 23, no.9, pp. 74-81, Sep. 2015.

[4] Z. Liu and L.-L. Yang, "Sparse or dense: a comparative study of codedomain NOMA systems," IEEE Trans. Wireless Commun.. Accepted for Publication.

[5] E. Hossain, M. Rasti, H. Tabassum, and A. Abdelnasser, "Evolution toward 5G multi-tier cellular wireless networks: an interference management perspective," IEEE Wireless Commun., vol. 21, no. 3, pp. 118-127, June 2014.

[6] M. Vameghestahbanati, I. D. Marsland, R. H Gohary and et al., "Multidimensional constellations for uplink SCMA systems-a comparative study," IEEE Commun. Survey \& Tutorials, vol. 21, no. 3, pp. 2169-2194, 2019.

[7] F. Wei, W. Chen, Y. Wu, and et al., "Toward 5G wireless interface technology: enabling nonorthogonal multiple access in the sparse code domain," IEEE Vehi. Tech. Mag., vol. 13, no. 4, pp. 18-27, Dec. 2018.

[8] L. Yu, P. Fan, D. Cai, and et al., "Design and analysis of SCMA codebook based on star-QAM signaling constellations," IEEE Trans. Vehi, Tech., vol. 67, no. 1, pp.10543-10553, Nov. 2018.

[9] Z. Mheich, L Wen, P. Xiao, and A. Maaref., "Design of SCMA codebooks based on golden angle modulation," IEEE Trans. Vehi, Tech., vol. 68, no. 2, pp.1501-1509, Feb. 2019.

[10] Z. Liu, P. Xiao, and Z. Mheich, "Power-imbalanced low-density signatures (LDS) from Eisenstein numbers," in The 16th IEEE Asia Pacific Wireless Communications Symposium (APWCS'2019), Singapore, August 2019, pp. 1-5.

[11] Z. Mheich, Z. Liu, P. Xiao, and A. Maaref, "Delayed bit interleaved coded sparse code multiple access," IEEE Trans. Vehi, Tech., vol. 69, no. 7, pp. 8018-8022, Jul. 2020.

[12] S. Maric, L. Z. Velimirovic, "Optimal frequency hopping patterns for FH-SCMA," Proc. in 2018 IEEE 5G World Forum (5GWF), Silicon Valley, CA, USA, 9-11 July 2018.

[13] Z. Bai, B. Li, M. Yang and et al., "FH-SCMA: frequency-hopping based sparse code multiple access for next generation internet of things," Proc. in IEEE Wireless Commun. Networking Conf. (IEEE WCNC 2017), San Francisco, USA, 19-22 March 2017.

[14] Q. Zeng, X. Liu, and P. Du, "Multi-level sequence-based frequencyhopping in multi-cell networks," IEEE Trans. Vehi, Tech., vol. 69, no. 12, pp. 16282 - 16287, Dec. 2020.

[15] D. Torrieri, S. Talarico, and M. Valenti, "Analysis of a frequencyhopping millimeter-wave cellular uplink," IEEE Trans. Wireless Commun., vol. 15, no. 10, pp.7089-7098, Oct. 2016. 